\title{
Seropositive Anti-NMDAR Mediated Autoimmune Encephalitis
}

\author{
Vykuntaraju K. Gowda ${ }^{1} \cdot$ Balamurugan Nagarajan ${ }^{1} \cdot$ Sanjay K. Shivappa ${ }^{2} \cdot$ Naveen Benakappa $^{2}$
}

Received: 19 January 2020 / Accepted: 16 April 2020 / Published online: 13 May 2020

(C) Dr. K C Chaudhuri Foundation 2020

To the Editor: Anti-N-methyl-D-aspartate receptor (AntiNMDAR) mediated autoimmune encephalitis is the most common cause of pediatric autoimmune encephalitis and literature on exclusively seropositive cases in pediatric population is scarce from India. Hence, this study was done to assess the clinicolaboratory profile and outcome of anti-NMDAR encephalitis. This is a retrospective chart review between January 2014 and August 2019. Children between 1 mo to 18 y with CSF NMDAR antibody positive were included. Clinical details, investigations, management and follow up details were analyzed.

Out of 55 cases of suspected autoimmune encephalitis, 23 $(42 \%)$ were serologically confirmed anti-NMDAR encephalitis; fourteen $(61 \%)$ were females. Clinical symptoms were insidious in onset in all children. Median age of onset of symptoms was $5 y$. Various clinical features seen were seizures $17(74 \%)$, involuntary movements $14(61 \%)$, speech disturbances $12(52 \%)$ and hemiparesis 5 (22\%). Behavioral changes, altered sensorium, sleep disturbances and autonomic dysfunction were seen in 15 $(65 \%)$ each. One child had epilepsia partialis continua. One child had preceding herpes simplex viral (HSV) encephalitis. MRI Brain was normal in 7 (30\%), cerebral atrophy 12 (52\%), mesial temporal signal changes $2(9 \%)$ and basal ganglia/thalamus and periventricular white matter signal changes in one child each. EEG revealed diffuse slowing in all and delta brush pattern in 14\%. Three (14\%) had CSF lymphocytic pleocytosis. All (96\%) children improved except one child who expired due to ventilator associated pneumonia. Follow up period ranged from a minimum of 3 mo to a maximum of 60 mo. Eighteen (78\%) reported either decreased scholastic performance or behavioral issues. Relapse was observed in $2(9 \%)$. Screening for tumors was negative in all.

Vykuntaraju K. Gowda drknvraju08@gmail.com

1 Department of Pediatric Neurology, Indira Gandhi Institute of Child Health, Bengaluru, Karnataka 560029, India

2 Department of Pediatric Medicine, Indira Gandhi Institute of Child Health, Bengaluru, Karnataka, India
In this study, clinical symptoms were insidious in onset in all children and this is comparable to Chakrabarty et al. [1]. Hemiparesis observed in $22 \%$ children in this study has not been reported in other pediatric case series [1-4]. Two children (8.6\%) suffered from relapse in this study, compared to $23 \%$ reported by Nagappa et al. [2]. However, other studies from India reported no relapses $[1,3,4]$. To conclude, pediatric NMDAR encephalitis presents as a sub-acute onset encephalopathy with extrapyramidal involvement, seizures, speech disturbance and autonomic involvement. Hemiparesis and epilepsia partialis continua could be the presenting feature of anti-NMDAR encephalitis. Early identification and prompt initiation of immunosuppressive therapy results in good outcome. Relapse can occur in pediatric age group.

Acknowledgements We would like to thank Dr. Anita Mahadevan, Professor, Department of Neuropathology, National Institute of Mental Health and Neuro-Sciences, Bengaluru for analysis and reporting of all CSF samples for autoimmune encephalitis panel for our patients.

\section{Compliance with Ethical Standards}

Conflict of Interest None.

\section{References}

1. Chakrabarty B, Tripathi M, Gulati S, et al. Pediatric anti-N-methylD-aspartate (NMDA) receptor encephalitis: experience of a tertiary care teaching center from North India. J Child Neurol. 2014;29: 1453-9.

2. Nagappa M, Bindu PS, Mahadevan A, Sinha S, Mathuranath PS, Taly AB. Clinical features, therapeutic response, and follow-up in pediatric anti-N-methyl-D-aspartate receptor encephalitis: experience from a tertiary care university hospital in India. Neuropediatrics. 2016;47:24-32.

3. Nair AV, Menon J, Kuzhikkathukandiyil P. Clinical profile and neuropsychiatric outcome in children with anti-NMDAR encephalitis. Indian Pediatr. 2019:56:247-9.

4. Suthar R, Saini AG, Sankhyan N, Sahu JK, Singhi P. Childhood antiNMDA receptor encephalitis. Indian J Pediatr. 2016;83:628-33.

Publisher's Note Springer Nature remains neutral with regard to jurisdictional claims in published maps and institutional affiliations. 